Цыдыпов Ш. Б., Герман Е. И., Жданов И. А. Семенова В. Э. Термостимулированные токи в неравновесных мелкодисперсных системах под действием внутренних полей

Научная статья

УДК: $537.9,536.425$

DOI 10.18101/2306-2363-2021-1-43-51

\title{
ТЕРМОСТИМУЛИРОВАННЫЕ ТОКИ В НЕРАВНОВЕСНЫХ МЕЛКОДИСПЕРСНЫХ СИСТЕМАХ ПОД ДЕЙСТВИЕМ ВНУТРЕННИХ ПОЛЕЙ
}

(C) Цыдыпов Ш. Б.

доктор технических наук, доцент

shulun@bsu.ru

(С) Герман Е. И.

старший преподаватель

net-admin@list.ru

(С) Жданов И. А.

аспирант кафедры общей и теоретической физики sqvan@mail.ru

(C) Семенова В. Э.

магистрант кафедры общей и теоретической физики evol_vs1994@mail.ru

Бурятский государственный университет имени Доржи Банзарова Россия, 670000, г. Улан-Удэ, ул. Смолина, 24a

\begin{abstract}
Аннотация. Исследованы токи термостимулированной деполяризации в гетерогенных системах из мелкодисперсных наночастиц диоксида углерода в одном случае, и механически измельченных частиц химико-лабораторного стекла в другом случае, помещенных в полярную водную матрицу. Выявлены закономерности спектров термостимулированных токов в рассматриваемых системах. Обсуждается природа пиков спектра и их взаимосвязь с процессами взаимодействия фаз в исследуемых системах.

Ключевые слова: термостимулированные токи, жидкость, стекло, спектр, фазовый переход, структура
\end{abstract}

\section{Для цитирования:}

Цыљыпов Ш. Б., Герман Е. И., Жданов И. А. Семенова В. Э. Термостимулированные токи в неравновесных мелкодисперсных системах под действием внутренних полей // Вестник Бурятского государственного университета. Химия. Физика. 2021. Вып. 1. С. 43-51.

В настоящее время заметное внимание уделяется исследования электрофизических свойств и структуры гетерогенных систем, получаемых погружением в полярную водную матрицу мелкодисперсных зерен различных твердых веществ с развитой поверхностью. Возникающие при этом разности потенциалов внутреннего электрического поля без подключения внешних источников тока, регистрируются высокочувствительными приборами для обнаружения электрическо- 
го тока. При нагревании гетерогенных систем величина этих токов испытывает ряд максимумов и минимумов, образуя спектр термостимулированных токов (TCT).

Известно, что свойства гетерогенных систем в основном определяются физико-химическим состоянием поверхности внедренных в матрицу частиц. Установление зависимости электрофизических свойств рассматриваемой системы от ее температуры, а также от электрической активности и удельной площади поверхности частиц является актуальной задачей в области флуктуационной электродинамики и физики поверхностных явлений.

На межфазных границах в рассматриваемой гетерогенной системе возникает кулоновское взаимодействие зарядов, расположенных на электрически активной поверхности внедренных частиц с полярными молекулами и ионами водной пленки. При наличии многочисленных границ раздела в исследуемых объектах суммарный эффект межфазных взаимодействий становится более сильным и является определяющим для процесса генерации внутренних электрических полей [1]. Источниками таких полей являются заряженные дефекты, локализованные на различных энергетических уровнях как поверхности, так и в объеме твердой компоненты, а также свободные ионы и полярные молекулы водной фазы [2].

Использование метода ТСТ позволяет получить информацию о состоянии структурных особенностей электрически активных систем, а также выяснить механизм межфазного взаимодействия их компонентов [3]. Исследование динамики процесса реализации термостимулированных токов при нагревании изучаемых объектов позволяет установить природу доминирующих в них релаксаторов и выявить условия разрушения дипольной поляризации [4].

Механоактивированные ультратонкие частицы характеризуются, как известно [5], высокой поверхностной концентрацией электрически активных дефектов, создающих локальное электрическое поле. В связи с этим происходит интенсивная адсорбция молекул воды на частицах, поле поверхности которых способно изменять свойства и структуру контактирующей с ними жидкости. Процесс адсорбции на мелкоразмерных твердых частицах под действием поля активных поверхностей его частиц можно рассматривать как последовательное наслоение молекул воды, что приводит в результате к образованию протяженных водных пленок из нескольких молекулярных слоев, которые по мере удаления от активных центров поверхности твердой фазы изменяют свою структуру и электрофизические свойства. Следует ожидать, что достаточно высокое содержание частиц с большой удельной поверхностью оказывает существенное влияние на процессы накопления и переноса заряда в рассматриваемой гетерогенной системе [6].

В данной работе исследуется гетерогенная система из мелкодисперсных наночастиц диоксида углерода в одном случае, и механически измельченных в ступе частиц химико-лабораторного стекла в другом случае, помещенных в полярную водную матрицу.

Образцы помещались между двумя плоскими, круглыми электродами диаметром 1,5 см, находящимися в керамической трубке, обмотанной снаружи нихромовой спиралью для регулируемого нагрева. С целью уменьшения теплового расширения эти электроды изготовлены из инвара и подключены к высокочув- 
Цыдыпов Ш. Б., Герман Е. И., Жданов И. А. Семенова В. Э. Термостимулированные токи в неравновесных мелкодисперсных системах под действием внутренних полей

ствительному вольтметр-электрометру В 7-49. Напряжение на электроды не подается. Поэтому, в эксперименте регистрировались токи, возникающие только под действием внутренних электрических и тепловых полей. Погрешность измерения тока составляла $10^{-15} \mathrm{~A}$

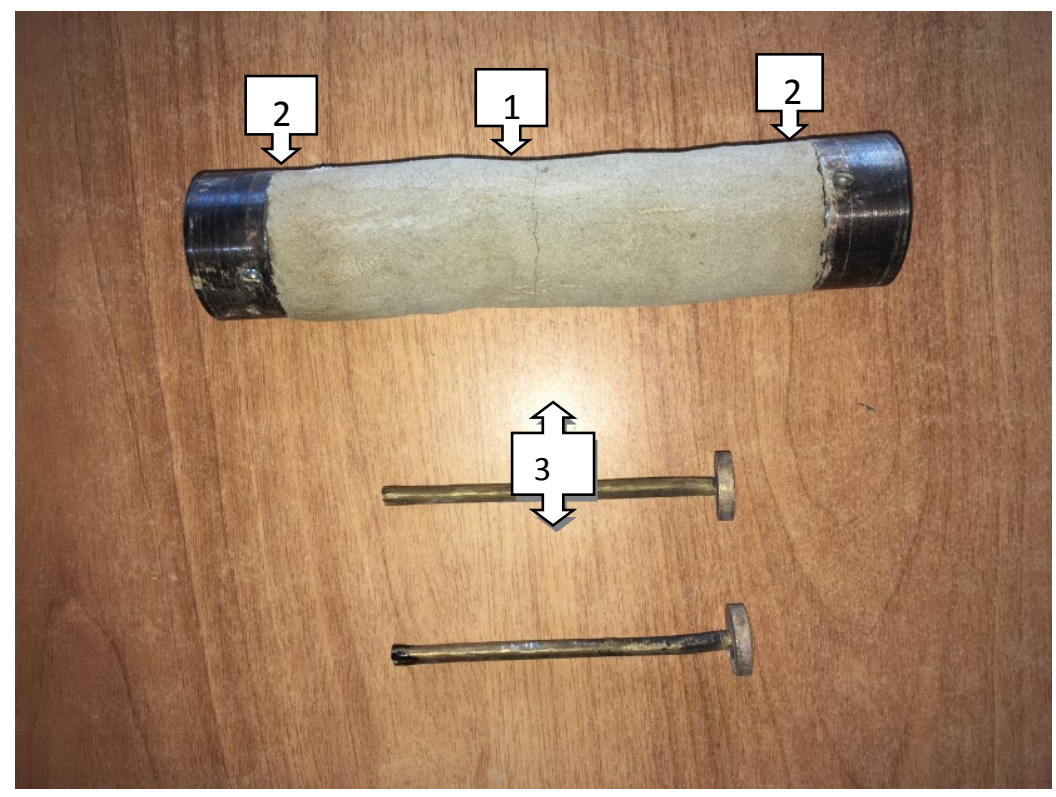

Рис. 1. Измерительная ячейка для измерения термостимулированных токов ТСТ. 1 Керамический цилиндр с обмоткой из нихромовой спирали, изолированной с помощью обмазки из глины, 2 - контакты спирали, 3 - электроды в виде поршня, изготовленные из инвара

Регистрация термостимулированных токов в исследуемых образцах производилась при нагреве образца с постоянной скоростью 1 град/мин. в интервале температур от 20 до $480^{\circ} \mathrm{C}$. Контроль температуры в измерительной ячейке производился с помощью терморегулятора REX-C100FK02-V*AN - SSR. Точность измерения температуры при этом составляла $0,5 \%$ в интервале $0-400^{\circ} \mathrm{C}$.

В связи с тем, что на электродах измерительной ячейки отсутствовало внешнее напряжение, динамика наблюдаемых спектров ТСТ зависела только от распределения собственных внутренних электрических полей, создаваемых локализованными носителями термоэлектретного заряда системы. Наблюдение динамики термостимулированных токов позволяет получить полную информацию о природе электретного состояния и его характеристиках в объекте исследования. Преимуществом метода ТСТ является возможность получения спектра энергии локализации зарядов в ловушках, что позволяет выявить структуру вещества и особенности его энергетического состояния. 


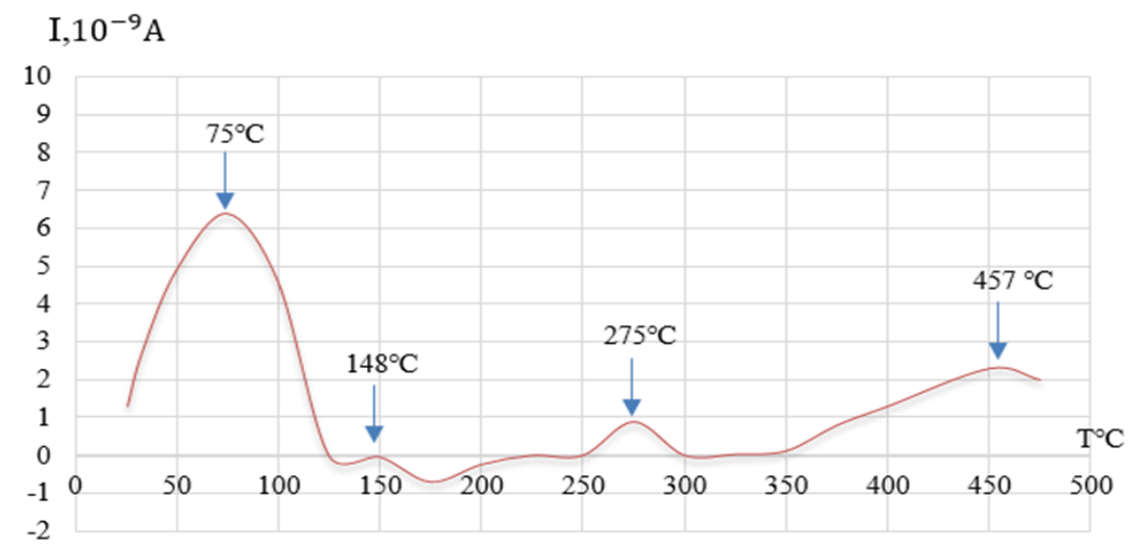

Рис. 1 Спектры термостимулированных токов для наноразмерного порошка диоксида кремния $\mathrm{SiO}_{2}$

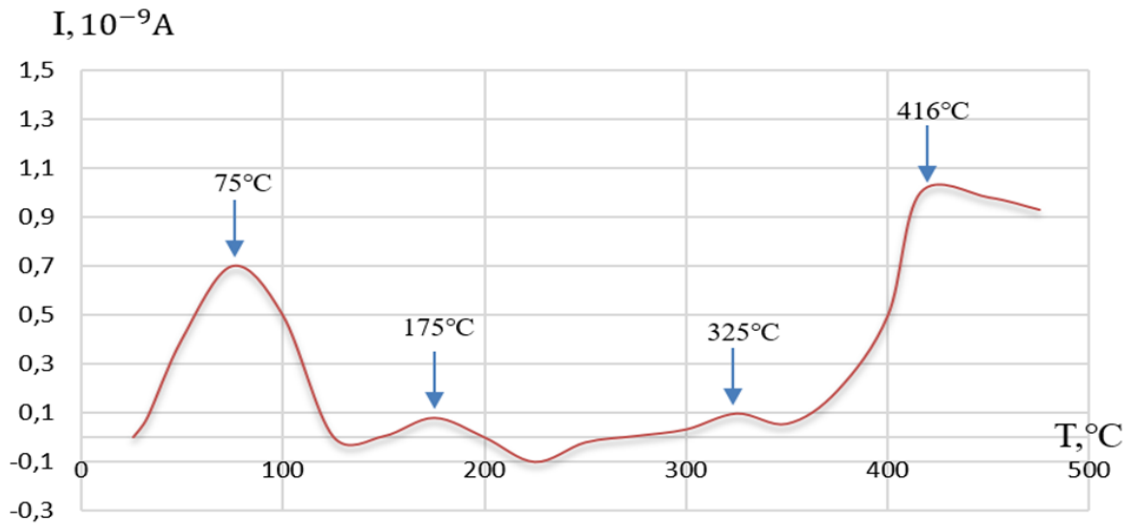

Рис. 2. Спектры термостимулированных токов для химико-лабораторного стекла

На рис. 1 и 2 представлены термограммы токов для нанопорошка диоксида кремния и механоактивированных мелкоразмерных частиц многокомпонентного химико-лабораторного стекла, погруженных в водную полярную матрицу. Полученные зависимости термостимулированных токов характеризуются наличием в исследуемом температурном интервале нескольких максимумов различной интенсивности и ширины. Это свидетельствует о наличии в исследуемой системе различных механизмов процессов переноса и релаксации зарядов вблизи границ раздела фаз. Каждый максимум тока на рис. 1 и 2 должен соответствовать разным типам электретных зарядов, образование которых зависит от температуры.

В табл. 1 приведены результаты анализа массовой доли химических элементов и компонентов химико-лабораторного стекла, проведенного на электронном растровом микроскопе JEOL6000 методом микрорентгеноспектрального анализа. 
Цыдыпов Ш. Б., Герман Е. И., Жданов И. А. Семенова В. Э. Термостимулированные токи в неравновесных мелкодисперсных системах под действием внутренних полей

Таблица 1

Массовые доли химических элементов и компонентов химико-лабораторного стекла

\begin{tabular}{|c|c|c|c|}
\hline Элемент & Содержание, \% & Соединение & Содержание, \% \\
\hline $\mathrm{Si}$ & 24,98 & $\mathrm{SiO}_{2}$ & 53,45 \\
\hline $\mathrm{Na}$ & 14,02 & $\mathrm{Na}_{2} \mathrm{O}$ & 3,93 \\
\hline $\mathrm{Mg}$ & 2,36 & $\mathrm{MgO}$ & 18,69 \\
\hline $\mathrm{Al}$ & 9,88 & $\mathrm{Al}_{2} \mathrm{O} 3$ & 18.91 \\
\hline $\mathrm{K}$ & 0,78 & $\mathrm{~K}_{2} \mathrm{O}$ & 0,95 \\
\hline $\mathrm{Ca}$ & 2,95 & $\mathrm{CaO}$ & 4,07 \\
\hline $\mathrm{O}$ & 45,03 & & \\
\hline
\end{tabular}

При низких температурах носителями заряда являются ионы жидкой полярной компоненты. Под действием собственного внутреннего поля двухфазной системы, происходит преимущественно ионизация полярных молекул воды, поскольку энергии теплового движения недостаточно для их ионизации. Согласно теории Пула-Френкеля, электрическое поле способствует уменьшению энергии ионизации полярных молекул. Это приводит к диссоциации молекул жидкой фазы и, связанным с ней, увеличением концентрации свободных ионов $\mathrm{n}_{\mathrm{i}}$

$$
n_{i}=n \exp \left(-\frac{U_{0}}{k T}\right) \exp \left(\frac{a \sqrt{E}}{k T}\right)
$$

где $\mathrm{n}$ - постоянная Лошмидта жидкой среды, $\mathrm{U}_{0}$ - энергия, необходимая для диссоциации молекул в отсутствие электрического поля, Е - напряженность внреннего поля, а — постоянная, зависящая от структуры жидкости.

При заданной температуре рассматриваемый заряд $\mathrm{Q}_{\mathrm{n}}$ релаксирует со средней скоростью, обратно пропорциональной времени релаксации. Температурная зависимость времени релаксации описывается в данном случае законом Арениуca $[7,8]$ :

$$
\tau_{n}=\tau_{0, n} \exp \left(\frac{U_{n}}{k T}\right)
$$

где $\tau_{\mathrm{n}}$ - время релаксации электретного заряда $\mathrm{n}-$ го типа, $\mathrm{U}_{\mathrm{n}}-$ его энергия активации, $\mathrm{T}$ - температура системы и $\tau_{0, \mathrm{n}}$ - предельное значение времени релаксации при бесконечно большой температуре. Из выражения (2) следует, что при низких температурах скорость релаксации рассматриваемого заряда и, следовательно, термостимулированные токи, сопровождающие данный процесс, имеют малую величину. При повышении температуры время релаксации уменьшается до постоянной величины $\tau_{0, n}$, характеризующейся только размером релаксирующего заряженного комплекса и частотой колебаний в удерживающей его потенциальной яме. Это приводит к наблюдаемой высокой интенсивности термостимулированных токов в некотором температурном интервале. Как известно [9, 10], профиль термостимулированного тока, обусловленного движением носителей заряда n-го типа, описывается выражением: 


$$
j\left(T, U_{0, n}, \tau_{0, n}, \beta\right)=-\frac{P_{0, n}}{\tau_{0, n}} \exp \left(-\frac{U_{n}}{k T}-\frac{1}{\beta \tau_{0, n}} \int_{T_{0}}^{T} \exp \left(-\frac{U_{n}}{k T^{\prime}}\right) d T^{\prime}\right)
$$

где $\mathrm{P}_{0}{ }^{(\mathrm{n})}$ - вектор поляризации исследуемой системы в начале ее линейного нагрева со скоростью $\beta, \mathrm{T}-$ температура системы, $\tau_{0}{ }^{(\mathrm{n})}-$ фактор релаксации, равный предельному значению времени релаксации $\tau_{\mathrm{n}}$ при достаточно высоких температурах и $\mathrm{U}_{\mathrm{n}}$ - энергия активации.

Анализ выражения (3) показывает, что при некоторой температуре $\mathrm{T}_{\mathrm{n}}$ термостимулированный ток достигает максимума, что позволяет определить энергию активации $U_{\mathrm{n}}$ носителей заряда, которая в данном случае является решением следующего трансцендентного уравнения:

$$
\frac{d}{d T}\left[\left.j\left(T, U_{n}, \frac{\beta U_{n}}{k T_{n}^{2}} \exp \left(\frac{U_{n}}{k T_{n}}\right), \beta\right)\right|_{T=T_{n}}=0\right.
$$

Время релаксации $\tau_{\mathrm{n}}$ при температуре максимума $\mathrm{T}_{\mathrm{n}}$ составляет

$$
\tau_{n}\left(T_{n}\right)=\frac{k T_{n}^{2}}{\beta U_{n}}
$$

Полная величина электретного заряда $\mathrm{Q}_{\mathrm{n}}$ носителей $\mathrm{n}$-го типа определяется как площадь под кривой зависимости термостимулированного тока от времени:

$$
Q_{n}=\int_{t_{1}}^{t_{2}} I(t) d t=\frac{1}{\beta} \int_{T_{n}^{(l)}}^{T_{n}^{(r)}} I(T) d T
$$

где $\mathrm{T}^{(\mathrm{l})}{ }_{n}$ и $\mathrm{T}^{(\mathrm{r})}{ }_{n}-$ нижняя и верхняя температурные границы существования этого заряда.

По спектрам ТСТ вычислены энергия активации носителей заряда

$$
U_{i}=\frac{k\left[T_{i, \max }\right]^{2}}{\Delta T_{i}}
$$

и плотности электретных зарядов

$$
\sigma_{i}=\frac{1}{S \beta} \int I(T) d T
$$

Положение пикового максимума тока $\mathrm{T}_{\mathrm{i}, \max }$ и его ширина $\Delta \mathrm{T}_{\mathrm{i}}$ определяются экспериментально, где $\mathrm{S}$ - площадь электрода; $\beta$ - скорость нагрева; T, K температура; I - величина тока; $\mathrm{k}$ - постоянная Больцмана; $\Delta \mathrm{T}_{\mathrm{i}}-$ ширина температурного интервала максимума термоактивационного тока.

Рассчитанные по спектрам ТСТ в соответствии с выражениями (7) и (8) энергии активации носителей заряда $\mathrm{U}_{\mathrm{n}}$ и плотности $\sigma_{\mathrm{n}}$, мелкодисперсного диоксида кремния $\mathrm{SiO}_{2}$ представлены в табл. 2. Полученные данные позволяют оценить релаксационные характеристики термостимулированных токов в изучаемой системе.

В спектрах ТСТ (рис. 1 и 2) можно выделить две области. В интервале низких температур $\left(20-80^{\circ} \mathrm{C}\right)$ наличие доминирующих максимумов тока при $\mathrm{T}=75^{\circ} \mathrm{C}$ на обоих рисунках может быть связано с накоплением межфазных гетерозарядов под действием внутреннего электрического поля, а также с особенностями структуры водной компоненты $[11,12]$. Экспериментально установлено, что 
Цыдыпов Ш. Б., Герман Е. И., Жданов И. А. Семенова В. Э. Термостимулированные токи в неравновесных мелкодисперсных системах под действием внутренних полей

температурное положение таких максимумов не зависит от концентрации полярной водной фазы, а также от структуры, размеров и химического состава твердой компоненты. Такие максимумы токов характерны как для неорганических, так и для органических дисперсных неупорядоченных систем $[11,12]$.

Таблица 2

Физические параметры электретного состояния наноразмерного порошка диоксида кремния

\begin{tabular}{|c|c|c|c|c|c|c|c|}
\hline $\begin{array}{l}\text { № } \\
\text { пика }\end{array}$ & $\mathrm{T}_{\text {max, }}{ }^{\circ} \mathrm{C}$ & $\begin{array}{l}\Delta \mathrm{T}, \\
{ }^{\circ} \mathrm{C}\end{array}$ & $\begin{array}{c}\mathrm{T}_{1},{ }^{\circ} \mathrm{C} \\
\text { Начало } \\
\text { пика }\end{array}$ & $\begin{array}{c}\mathrm{T}_{2},{ }^{\circ} \mathrm{C} \\
\text { Конец } \\
\text { пика }\end{array}$ & $\begin{array}{l}\mathrm{I}, \mathrm{A} \\
10^{-10}\end{array}$ & $\mathrm{U}$, эB & $\sigma, \mathrm{Kл} / \mathrm{M}^{2}$ \\
\hline 1 & 75 & 99 & 26 & 125 & 0,340 & 0,028 & $3,74 \cdot 10^{-4}$ \\
\hline 2 & 148 & 48 & 127 & 175 & 0,003 & 0,0047 & $4,1 \cdot 10^{-7}$ \\
\hline 3 & 275 & 49 & 250 & 299 & 0,089 & 0,08 & $3,15 \cdot 10^{-5}$ \\
\hline 4 & 457 & 25 & 350 & 475 & 0,290 & 0,15 & $2,67 \cdot 10^{-5}$ \\
\hline
\end{tabular}

На границе контакта водной компоненты с электрически активной поверхностью низкоразмерных частиц происходит накопление свободных зарядов. Источником таких зарядов являются процессы диссоциации в полярной жидкой матрице системы, в ходе которых молекулы воды распадаются на ионы. Согласно теории Пула-Френкеля, диссоциация молекул жидкости в окрестности температуры $75^{\circ} \mathrm{C}$ происходит преимущественно под действием внутреннего электрического поля, поскольку величина энергии теплового движения молекул недостаточна для их ионизации.

Таблица 3

Физические параметры электретного состояния мелкодисперсного химико-лабораторного стекла

\begin{tabular}{|c|c|c|c|c|c|c|c|}
\hline $\begin{array}{c}\text { № } \\
\text { пика }\end{array}$ & $\mathrm{T}$ max, ${ }^{\circ} \mathrm{C}$ & $\Delta \mathrm{T},{ }^{\circ} \mathrm{C}$ & $\begin{array}{c}\mathrm{T}_{1},{ }^{\circ} \mathrm{C} \\
\text { Начало } \\
\text { пика }\end{array}$ & $\begin{array}{c}\mathrm{T}_{2},{ }^{\circ} \mathrm{C} \\
\text { Конец } \\
\text { пика }\end{array}$ & $\begin{array}{c}\mathrm{I}, \mathrm{A} \\
10^{-10}\end{array}$ & $\mathrm{U}$, эB & $\sigma, \mathrm{Kл} / \mathrm{M}^{2}$ \\
\hline 1 & 75 & 99 & 26 & 125 & 0,07 & 0,028 & $4 \cdot 10^{-4}$ \\
\hline 2 & 175 & 51 & 149 & 200 & 0,008 & 0,0053 & $1,24 \cdot 10^{-6}$ \\
\hline 3 & 325 & 59 & 290 & 349 & 0,0098 & 0,0092 & $2,03 \cdot 10^{-6}$ \\
\hline 4 & 416 & 100 & 350 & 450 & 0,1 & 0,1 & $5,96 \cdot 10^{-5}$ \\
\hline
\end{tabular}

Накопление межфазного заряда и характерное время его релаксации обусловлены Максвелл-Вагнеровским механизмом поляризации $[11,12]$. Эти параметры связаны с содержанием водной компоненты в исследуемой системе и активностью поверхности входящих в ее состав мелкоразмерных механоактивированных частиц. При низких температурах находящиеся в водной пленке заряды удерживаются, преимущественно, вблизи активных центров поверхности твердых частиц, которые создают потенциальный барьер, препятствующий свободному движению захваченных зарядов. Регистрация токов в системе осуществля- 
ется только вследствие наличия в ней градиентов потенциала внутренних собственных электрических полей, свободных ионов и каналов ионной проводимости.

Эффективность кластеризации структуры полярной воды напрямую связана с активностью поверхности твердой фазы, с которой жидкая полярная фаза взаимодействует. Подтверждением эффективности межфазного взаимодействия является также увеличение плотности заряда, вычисленной по профилю термостимулированного тока.

Максимум тока при повышенных температурах проявляет зависимость от величины адсорбции и от поверхностной активности мелкоразмерных частиц. Появление этих пиков связано, по всей вероятности, с термическим разрушением и освобождением ионов из дефектных областей, расположенных на поверхности и в объеме частиц твердой фазы. Значительная амплитуда максимума тока свидетельствует о большой концентрацией накопленных заряды на межфазных границах.

\section{Литература}

1. Mascarenhas S. The electret effect in bone and biopolymers and the bound water problem // Ann. N Y. Acad. Sci. 1974. V. 238. P. 36-52.

2. Гороховатский Ю. А., Бордовский Г. А. Термоактивационная токовая спектроскопия высокоомных полупроводников и диэлектриков. Москва: Наука, 1991. 248 с.

3. Тареев Б. М. Физика диэлектрических материалов. Москва: Энергоиздат, 1982. $320 \mathrm{c}$.

4. Groenink J.A., Binsma H. H. Electrical conductivity and defect chemistry of $\mathrm{PbMoO}_{4}$ and $\mathrm{PbWO}_{4} / /$ J. Sol. Stat. Chem. 1979. V. 29, № 2. P. 227-238.

5. Танаев А. Б., Щербаченко Л. А., Безрукова Я. В., Цыдыпов Ш. Б. и др. Особенности процессов накопления и транспорта электретных зарядов в мелкоразмерных разупорядоченных структурах под действием внутреннего напряжения // ЖТФ. 2017. Т. 87, № 3. С. 383-389.

6. Бузунова М.Ю., Цыдыпов Ш.Б., Щербаченко Л.А. и др. Перенос электретных зарядов в неравновесных мелкодисперсных системах под действием внутреннего поля // Вестник Бурятского государственного университета. Физика. Химия. 2015. № 3. С. 7580.

7. Groenink J. A., Binsma H. Electrical conductivity and defect chemistry of $\mathrm{PbMoO}_{4}$ and $\mathrm{PbWO}_{4}$ // J. Sol. State Chem. 1979. V. 29, № 2. P. 227-236.

8. Электреты: сборник / под ред. Г. Сесслера; пер. с англ. А. Ю. Гросберга. Москва: Мир, 1983. 487 с.

9. Пинчук Л. С., Кравцов А. Г., Зотов С. В. Термостимулированная деполяризация крови человека // ЖТФ. 2001. Т. 71, Вып. 5. С. 115-118.

10. Карнаков В. А., Ежова Я. В., Марчук С. Д., Харлан А. Р., Щербаченко Л. А. Термоактивационная спектроскопия тонких прослоек воды // Изв. ВУЗов. Физика. 2008. T. 51, № 8. С. 57-60.

11. Борисов В. С., Карнаков В. А., Ежова Я. В., Рубцова О. Б., Щербаченко Л. А. Особенности поляризации тонких пленок воды в поле активной поверхности кристалла слюды // ФТТ. 2008. Т. 50, Вып. 6. С. 980-985.

Статья поступила в редакцию 14.11.2020; одобрена после рецензирования 21.01.2021; принята к публикации 29.01.2021 
Цыдыпов Ш. Б., Герман Е. И., Жданов И. А. Семенова В. Э. Термостимулированные токи в неравновесных мелкодисперсных системах под действием внутренних полей

THERMOSTIMULATED CURRENTS IN NON-EQUILIBRIUM FINELY DISPERSED SYSTEMS UNDER THE ACTION OF INTERNAL FIELDS

Tsydypov Sh. B.

Doctor of Technical Sciences, Professor

Buryat State University

shulun@bsu.ru

German E. I.

Senior Lecturer

Buryat State University

net-admin@list.ru

Zhdanov I. A.

Postgraduate student

Department of General and Theoretical Physics, Buryat State University

sqvan@mail.ru

Semenova $V$. . E.

Master's student

Department of General and Theoretical Physics

evol_vs1994@mail.ru

Abstract. Investigation were thermostimulated depolarization currents in heterogeneous systems of finely dispersed nanoparticles of carbon dioxide in one case, as well as mechanically crushed particles of chemical laboratory glass in other case, placed in a polar water matrix. The regularities of the spectra of thermostimulated currents in the systems under consideration were revealed. The nature of the spectrum peaks and their relationship with the processes of phase interaction in the systems under study are discussed.

Keywords: thermostimulated currents, liquid, glass, spectrum, phase transition, structure

For citation

Tsydypov Sh. B., German E. I., Zhdanov I. A., Semenova V. E. Thermostimulated Currents in Non-Equilibrium Chalk-Codisperse Systems Under the Action of Internal Fields // Bulletin of Buryat State University. Chemistry. Physics. 2021; 1: 43-51 (In Russ.).

The article was submitted 14.11.2020; approved after reviewing 21.01.2021; accepted for publication 29.01.2021. 